\title{
A Prospective Randomized Study to compare Levobupivacaine and Ropivacaine in Patients Undergoing Forearm Orthopaedic Surgeries under Ultrasound-Guided Supraclavicular Brachial Plexus Block
}

\author{
${ }^{1}$ WASIM KHURSHEED MIR, ${ }^{2}$ VIPIN KUMAR VARSHNEY, ${ }^{3}$ MD SHAHBAZ ALAM, ${ }^{2}$ RATEEK SINGH, ${ }^{4}$ G.S. JHEETAY, \\ ${ }_{1}^{1}$ ADITYA SAMUDRALA \\ ${ }^{1}$ Junior Resident, Department of Anaesthesia, Teerthanker Mahaveer Medical College \& Research Centre, TMU, Moradabad, India. \\ ${ }^{2}$ Assistant Professor, Department of Anaesthesia, Teerthanker Mahaveer Medical College \& Research Centre, TMU, Moradabad, India. \\ ${ }^{3}$ Associate Professor, Department of Anaesthesia, Teerthanker Mahaveer Medical College \& Research Centre, TMU, Moradabad, India. \\ ${ }^{4}$ Professor \& HOD, Department of Anaesthesia, Teerthanker Mahaveer Medical College \& Research Centre, TMU, Moradabad, India. \\ Corresponding Author: Dr. Prateek Singh, Assistant Professor, Department of Anaesthesia, Teerthanker Mahaveer Medical College \& \\ Research Centre, TMU, Moradabad, India.dralamshahbaz006@gmail.com
}

\begin{abstract}
Background: Brachial plexus block utilizing ultrasound imaging has now become either adjuvant to general anaesthesia (GA) or as a mainstay anaesthesia modality. There are fewer studies comparing the effects of ropivacaine and levobupivacaine for supraclavicular brachial plexus block. The aim of this study was to do a prospective randomized study to compare $0.5 \%$ Levobupivacaine and $0.5 \%$ Ropivacaine in patients undergoing forearm orthopaedic surgeries under Ultrasound-Guided Supraclavicular Brachial Plexus Block.

Materials and Methods: A total of 56 patients were enrolled and randomized into two groups. Group R with 28 patients was given $30 \mathrm{~mL}$ of $0.5 \%$ Ropivacaine and Group $\mathrm{L}$ with 28 patients were given $30 \mathrm{~mL}$ of $0.5 \%$ Levobupivacaine, drugs were used for giving supraclavicular block under ultrasound. Parameters assessed were onset and duration of sensory and motor block, duration of analgesia, and any adverse events. After administration of block, the block characteristics were assessed every $5 \mathrm{mins}$ till the onset of the complete blockade, then hourly till the effect of the block. Data between the groups were analysed using SPSS 25.0 software.

Results: Demographically both the groups were comparable in the study. The study shows that there was a statistically significant difference in onset of sensory block in Levobupivacaine and Ropivacaine (7.54 mins \pm 2.10 vs 8.55 mins \pm 2.08 ), similarly there was the difference in onset of motor block in Levobupivacaine 12.95 mins \pm 2.30 vs Ropivacaine 14.07 mins \pm 2.22 . The duration of analgesia was more in the group of patients Levobupivacaine (9.98 hours \pm 4.88 ) Ropivacaine (8.03 hours \pm 3.58 )

Conclusion: the onset of action of sensory and motor was early in Levobupivacaine group with faster recovery of motor function as compared to the equivalent dose of Ropivacaine. Levobupivacaine has a better profile in terms of duration of analgesia.

Keywords: Brachial plexus block, ropivacaine, levobupivacaine, supraclavicular brachial plexus block, ultrasound guidance
\end{abstract}

\section{INTRODUCTION}

The Peripheral nerve plexus blocks have a dominant role in current anaesthesia working since they provide adequate conditions for operation without any significant adverse effects.[1] This technique is advantageous in cases of upper limb orthopaedic as well as reconstructive surgeries, and in emergency surgeries. The most enchanting part of the blocks is not only there ease intraoperative but also reliable postoperative analgesia. [2]

The regional nerve block is advantageous as it is cost-effective, with adequate postoperative analgesia without the complications of general anaesthesia. These days with the advent of technology nerve localization is commonly done by using peripheral nerve stimulator or using ultrasound guidance $[3,4]$

Brachial plexus block is a technique from the 19th century, which has now become either adjuvant to general anaesthesia (GA) or as a mainstay anaesthesia modality. Orthopaedic surgeries if performed on time gives the best results saving the limb of the patient thus not letting the quality of life to get to deteriorated, peripheral nerve blocks can be used in patients with significant comorbidities without the added risks of GA. [5-7]
With the advent of USG imaging, vital structures in the supraclavicular region can be easily identified in realtime along with optimum local anaesthetic spread.[8]

Ropivacaine and Levobupivacaine are propyl homologues of Bupivacaine. They have low lipid solubility, short elimination half time, higher plasma clearance, lesser affinity to cardiac tissues than parent drug Bupivacaine. Levobupivacaine is also a safe and effective local anaesthetic drug for spinal and epidural anaesthesia.[9]

This prospective randomized study was done to compare Levobupivacaine and Ropivacaine in patients undergoing forearm orthopaedic surgeries under Ultrasound-Guided Supraclavicular Brachial Plexus Block.

\section{MATERIALS AND METHODS}

After obtaining approval from Institutional Ethics Committee, this clinical study entitled "A Prospective Randomized Study to compare Levobupivacaine and Ropivacaine in Patients Undergoing Forearm Orthopaedic Surgeries under Ultrasound-Guided Supraclavicular Brachial Plexus Block" was carried out in the Department of Anaesthesiology, Teerthanker Mahaveer Medical College and Research Centre, Moradabad. 
The CTRI registration no - CTRI/2019/04/018608.

The study population has been calculated by using Formula $((Z \times Z) \alpha / 2 \quad(S D) \times(S D) / E \times E)[10]$ which comes nearly total of 56 patients of ASA grade I and II, which was randomised into two separate groups of 28 patients each by closed envelope technique. One group will receive $30 \mathrm{ml}$ of $0.5 \%$ of Levobupivacaine another group will receive $30 \mathrm{ml}$ of $0.5 \%$ of Ropivacaine.

Inclusion criteria for the patients were - Written informed consent given, American society of anaesthesiologists (ASA) grade I\& II patients, age group 18- 65 years, patients with BMl $18.5-22.9 \mathrm{~kg} / \mathrm{m} 2$, elective cases. Exclusion criteria [11] consists of infection at the end site of injection, neuromuscular disorder, patients with pre-existing diseases, cardiovascular or cerebrovascular diseases, severely altered mental status, the patient converted to general anaesthesia intra-operatively.

Complete history and physical examination (Neurological examination of the upper limb for any nerve damage has to be specifically ruled out) with appropriate investigations was done for every patient one day prior. All the patients were kept nil orally for at least 6 hours before the procedure. An intradermal sensitivity test of Ropivacaine and Levobupivacaine was performed the night before. Inj. Ondansetron $4 \mathrm{mg}$ I.V was given 30 minutes before operation. Intravenous ringer lactate fluid was started with $20 \mathrm{G}$ cannula. In the Operation theatre monitors were attached (Non-Invasive Blood pressure, ECG, SpO2) and patients were placed in a supine position, with the head turned away and the ipsilateral arm adducted. The skin was prepared with $10 \%$ povidoneiodine solution. All the patients received nerve block using ultrasound (Sono site M-Turbo, Sono site, Inc., Bothwell, $\mathrm{MO}$, USA) guided probe (frequency 10-15 MHz, covered with a sterile dressing) which was used to locate subfascial intracluster of supraclavicular brachial plexus.[7] The transducer was positioned just superior to the clavicle in its midpoint, with a slight caudal tilt to visualize the crosssectional image of subclavian artery, the nerve bundles will appear as hypoechoic oval structure just superior and posterior to the subclavian artery. Using a 25 gauze needle $1.5 \mathrm{ml}$ of local anaesthetic was injected just lateral to the probe in order to avoid the pain during needle insertion. Under direct visualization, the local anaesthetic was given in small increments as per the groups allocated after adequate aspiration. After removal of needle digital pressure with gauze, the piece was applied at the site for 5 min. Sensory and motor block were evaluated preoperatively to determine a baseline and every 5 min for $30 \mathrm{~min}$ or until the onset of the blockade was noted and thereafter every $60 \mathrm{~min}$. The sensory block was assessed by the pinprick method (22G hypodermic needle). Assessment of sensory block was done in the dermatomal areas corresponding to the median nerve, radial nerve, ulnar nerve, and musculocutaneous nerve till complete sensory blockade was achieved. Sensory onset was considered when there was a dull sensation to pinprick along with the distribution of any of the above-mentioned nerves.[8]A complete sensory block was considered when there was a complete loss of sensation to pinprick. Sensory block was graded as- Grade 0: Sharp pain, Grade 1: Analgesia, dull sensation, Grade 2: Anaesthesia, no sensation. A modified Bromage Scale[9] for the upper extremity was used to assess the motor function. This scale consists of the following four scores- Score 0 - Able to raise the extended arm to 90 degrees for a full $2 \mathrm{sec}$, Score 1 Able to flex the elbow and move the fingers but unable to raise the extended arm. Group 2 - Unable to flex the elbow but able to move the fingers Group 3 - Unable to move the arm, elbow, or fingers. The onset of motor blockade was considered when there was a Grade 1 motor blockade. Peak motor block was considered when there was a Grade 3 motor blockade. Block was considered to have failed when sensory anaesthesia was not achieved within 30 min. General anaesthesia was given subsequently to these patients who were then excluded from the study. Hemodynamic parameters and vitals (Blood pressure, Heart rate, Respiratory rate, and Oxygen saturation) were also monitored during the procedure. Duration of analgesia was assessed by using a 10 point Visual Analogue Scale (VAS)[11] in which a score of "0" indicates "no pain" and a score of "10" "worst pain imaginable". The VAS measurements were obtained every 5 minutes for 30 minutes initially and then hourly till the score of 4 . The rescue analgesia in the form of injection diclofenac sodium $(1.5 \mathrm{mg} / \mathrm{kg}$ ) intramuscularly was administered at the Visual Analogue Scale score of $\geq 4$.Duration of sensory block was determined by noting the time when there was a return of dull sensation to pinprick and duration of motor blockade was determined by noting the time the patients could first move their fingers.[12]The side-effects such as bradycardia, hypotension, pruritus, and respiratory distress were looked for.

\section{STATISTICAL ANALYSIS}

The data of total 56 patients were taken and each group consist of at least 28 patients with an $\alpha$ error $=0.05$, power $=80 \%$. Results were accepted with a $p$-value of $<0.05$. Statistical analysis was performed using Statistical Package for Social Science software version 25.0. (SPSS Inc, Chicago, IL, USA). The student t-test was used for comparing the mean values of the continuous variables between the two groups. The chi-square test was applied for comparing the categorical variables such as gender, adverse events between the two groups.

\section{RESULTS}

The present study was aimed to compare 56 patients for the efficacy of Levobupivacaine and Ropivacaine for brachial plexus block undergoing orthopaedic surgeries in the upper limb.

Consort diagram of the study is shown in Figure 1.

The demographic profile of the patient's age, sex, ASA grade were comparable in both groups. (Table 1) Hemodynamic variables were comparable with respect to heart rate, systolic, diastolic and mean blood pressure in both the groups. (Figure 2) There was no significant difference in mean respiratory rate and mean oxygen saturation in both groups. The onset of mean sensory and motor blockade was significantly less in group Levobupivacaine. (Table 1) Distribution of both mean sensory and motor blockade with respect to time was analysed. (Table 2 and 3). Duration of pain-free analgesic time was higher more among Levobupivacaine against 
Ropivacaine. The mean VAS scoring and analgesic duration are depicted in Figure 3. No complications were reported among both Levobupivacaine and Ropivacaine groups. All the blocks were successfully performed.

\section{DISCUSSION}

Regional nerve block brachial nerve plexus, today popularly conducted by the use of ultrasound technology, nowadays it's standard for the regional surgeries.[13] Brachial plexus blocks can be administered via three approaches - inter scalene groove, supraclavicular, infraclavicular and axillary. [14] However, nowadays progress in ultrasound machines have marked the interests of perioperative physicians to use supraclavicular anaesthesia delivery approach most often because of realtime visualization of pleura.[15] The brachial plexus is surrounded around the 1st rib posteriorly and superficial to subclavian artery. The utilization of ultrasound for regional anaesthetic technique gives a visual arrangement of tissues such as lung surface and arteries with direct picturing the spread of local drugs by the antiseptic needle to provide anaesthesia.[16]

Benefits for regional anaesthesia are excellent perioperative and post-procedure pain-free time, avoidance of opioid-related side effects decreased recovery time and short hospital stay. Local anaesthetic agent choice, dose, volume, concentration and use of adjuncts govern the onset, extent, quality and duration of anaesthesia. Ropivacaine and Levobupivacaine being less lipophilic generally block only the small A-delta and $\mathrm{C}$ conduction fibre responsible for sensory block and large myelinated Aa fibres are usually spared, thus reporting lesser motor block than Bupivacaine. This property of these enantiomers makes them less efficacious in joining the cardiac sodium channels thus rendering them less cardiotoxic too.[16-18]

In the current study, the mean age in both groups Levobupivacaine is 30.93 and Ropivacaine is 31.14 . The gender distribution in Levobupivacaine is $78 \%$ are males and $21 \%$ are females whereas in Ropivacaine it's $67.9 \%$ males and $32.1 \%$ females. Ropivacaine group 17 patients had ASA grade 1 and 11 patients had ASA grade 2 .

Contrarily findings were observed at Garg et al[16] reported comparable between the two groups. Patients in group $\mathrm{L}$ had a mean age of 55.75 years (Range: 2364years) and those in group $R$ had mean age 52.35 years (Range: $24-62$ years). In group L, $8(40 \%)$ patients were males and $12(60 \%)$ females, whereas in group R, $11(55 \%)$ patients were males and $9(45 \%)$ patients were females. As per ASA grade, in group $L 7(35 \%)$ patients were of grade I and $13(65 \%)$ patients belonged to grade II. In group $\mathrm{R}$, $5(25 \%)$ patients were grading me and $15(75 \%)$ patients belonged to grade II.

The statistically significant difference in onset of the blockade in sensory (7.54 \pm 2.10$)$ and motor (12.95 \pm 2.30$)$ with specifics for mean was observed faster to the subjects who received compound Levobupivacaine versus patients of group $\mathrm{R}$ opivacaine with sensory(8.55 \pm 2.08$)$ and motor(14.07 \pm 2.22$)$. Similar results were observed by Kulkarni et al[1] and Mageswaran and Choy.[19] Similarly in the study conducted by Deshpande et al[20], in their study, they observed that the onset of sensory and motor block was earlier with levobupivacaine $0.5 \%$ with a statistically higher significance.

The duration of sensory, motor block and postoperative analgesia(9.98 \pm 4.88$)$ was prolonged with $0.5 \%$ levobupivacaine as compare to the analgesic effect of $0.5 \%$ ropivacaine $(8.03 \pm 3.58)$ in supraclavicular brachial plexus block.[21] Garg et al[16] disclosed that comparable onset time for sensory (group L: $5.36 \pm 1.18$ minutes; group $\mathrm{R}$ : $5.74 \pm 1.25$ minutes) and motor blockade (group L: 7.11 \pm 1.07 minutes; group $R: 7.62 \pm 1.23$ minutes) which was in coincident with findings of Casati et al[22] who showed $30 \mathrm{~mL}$ of levobupivacaine group drug have an inter scalene block of similar start time as the onset by the Ropivacaine (20 min; $p=0.53$ ), similar findings were reported by Liisanantti et al.[23]

These findings are coincidental with this study, in which $0.5 \%$ levobupivacaine mean onset of sensory \& motor block $7.54 \pm 2.10$ and $12.95 \pm 2.30$ with a p-value of 0.045 and ropivacaine $8.55 \pm 2.08$ and $14.07 \pm 2.22$ with a $p$ value of 0.048 . The onset time was quicker in the current study compared to other studies because the drugs were deposited directly near nerves under ultrasound guidance.

An opposite trend in the paper of Mankad et al[14] \& Gonzalez et al[24] was seen as they found a faster onset of sensory and motor blockade for Ropivacaine than Levobupivacaine which was statistically significant because most anaesthetic agents block $C$ fibres at the same rate but a fibre blockade depends on the chemical properties of the drug and also the difference in the anatomical location of nerve blocks and the technique used.

Rathore and Bhosale[15] found that in-group R, mean required to the sensory block $(8.24 \pm 2.26)$ mins in the start as compared to $(10.6 \pm 3.19)$ mins in group $L(p<0.05$ value). Mankad et al[14] observed non-informative points in the sensory block, in this analysis anaesthesia for nerves for the motor was notably brisk with drug $R$ ( $9.50 \pm 2.403$ min )as juxtapose to drug $L(12.33 \pm 2.537 \mathrm{~min})$.

In this study, the mean VAS score at 15 minutes was significantly more among Levobupivacaine $(0.18 \pm 0.48)$ compared to Ropivacaine $(1.39 \pm 0.83)$ with a $p$-value of 0.001 . In a study done by Kulkarni et al[1] stated that the VAS was less in Levobupivacaine which was found to be statistically significant especially from 8th hour onwards.

Garg et al[16] stated that in the postoperative period, VAS scores were analysed, scores were comparable until 6 hours postoperatively, beyond 6 hours the VAS scores were comparably lower in Levobupivacaine group at 6 hours and at 8 hours. Cline et al[13] too reported lower VAS for Levobupivacaine after 8 hours postoperatively but was comparable. Gonzalez et al[24] reported higher VAS for Ropivacaine than Levobupivacaine at the time of the first analgesic request.

The painless duration was more in Levobupivacaine $(9.98 \pm 4.88)$ compared to Ropivacaine $(8.03 \pm 3.58)$. [23] the total pain-relief is 9.98 hours in drug group Levobupivacaine vs 8.03 hours in group Ropivacaine. Casati et al[22] reported that there was no difference in postoperative pain scores comparing levobupivacaine and ropivacaine. Chauhan et al[25] found that VAS score was 0 up to 5 hours in group $L$ and 2 hours in group $R$. The VAS score of $\geq 4$ was attained in 4 hours in Group $R$ and by 9 hours in group $L$. 
In a study conducted by Gonzalez-Suarez et al.,[24] the duration of analgesia was seen to be prolonged with ropivacaine $(11.3 \pm 4.1 \mathrm{~h})$ than with levobupivacaine $(9.2 \pm$ $3.1 \mathrm{~h})$, which was inverse than our study.

Hemodynamically there were no significant observations in the study. All the parameters (Mean PR, SBP, DBP, MAP, SPO2) were found to be statistically insignificant. Similar findings were found in studies performed by Kulkarni et al[1] stated that mean heart rate, systolic and diastolic blood pressure for both the groups were compared postoperatively and observed no statistically significant difference among the groups. Similar results related to haemodynamic parameters were found in a study conducted by Deshpande et al and found that there was no significant difference between both the group's heart rate and blood pressure, ECG and SPO2 were maintained throughout the surgery.[20] The same findings are also observed by Fusun et al.[26]

In this study, the patients were observed for any mishap. In both the groups no patient-reported for any unsuccessful brachial plexus block. No complications had been reported, this was similar to the study by Kulkarni et al.[1]

The decreased toxicity of levobupivacaine is attributed to its $S$ type enantiomer and faster protein binding rate which is alike to Ropivacaine.

In a similar study conducted by Chauhan et al [25] found that there was no significant incidence of complications in either group. Similarly, Mankad et al [14] also observed that there were also no drawbacks.

The regional anaesthesia administered with the aid of ultrasound technology hypothetically prevents inadvertent intraneural and intravascular injections, but the evidence needs thorough investigations. Ropivacaine offers an advantage where early recovery of motor function is desired in the postoperative period as compared to motor recovery profile of Levobupivacaine. Levobupivacaine has a better profile in terms of onset of block, duration of analgesia, with a considerable disadvantage of delayed wearing off of the motor blockade. Levobupivacaine should be considered when postoperative analgesia is a concern but not when the early return of motor activity is required.

\section{CONCLUSION}

The onset of action of sensory and motor is acceptable in the ropivacaine group as it has a faster recovery of motor function as compared to an equivalent dose of levobupivacaine. Ropivacaine offers an advantage where early recovery of motor function is desired in the postoperative period as compared to motor recovery profile of levobupivacaine. Levobupivacaine has a better profile in terms of duration of analgesia, with a considered disadvantage of delayed wearing off of the motor blockade. Levobupivacaine should be considered when postoperative analgesia is a concern but not when the early return of motor activity is required.

\section{REFERENCES}

1. Kulkarni SB, Pimpare M, Balasaheb T, Govardhane. Comparison of levobupivacaine with Ropivacaine for supraclavicular brachial plexus block. Int J Res Med Sci. 2016;4:3789-96
2. Damien B, Murhy, Collin JL, Cartney, Vincent WS. Novel analgesic adjuvants for brachial plexus block: A Systemic review. Anesth Analg. 2000;90:1122-8.

3. Abrahams MS, Aziz MF, Fu RF, Horn JL. Ultrasound guidance compared with electrical neurostimulation for peripheral nerve block: A systematic review and metaanalysis of randomized controlled trials. $\mathrm{Br} \mathrm{J}$ Anaesth. 2009;102:408-17.

4. Williams SR, Chouinard P, Arcand G, Harris P, Ruel M, Boudreault $D$, et al. Ultrasound guidance speeds execution and improves the quality of supraclavicular block. Anesth Analg. 2003;97:1518-23.

5. Kumar S, Palaria U, Sinha AK, Punera DC, Pandey V. Comparative evaluation of ropivacaine and ropivacaine with dexamethasone in supraclavicular brachial plexus block for postoperative analgesia. Anesth Essays Res. 2014;8:20208 .

6. Kunitaro W, Joho T, Alan KL, Kumi M, Hideaki S, Tetsuo I, Tomoko Y. Postoperative analgesia comparing levobupivacaine and ropivacaine for brachial plexus block: $A$ randomized prospective trial. Med (Baltimore). 2017;96:6457.

7. Macfarlane A, Perlas A, Chan V, Brull R. Eight ball, corner pocket ultra-sound-guided supraclavicular block: avoiding a scratch. Reg Anesth Pain Med. 2008;33:502-3.

8. Dilip K, Supraclavicular brachial plexus block: A new approach. Indian J Anaesth. 2003;47:287-88.

9. Brull R, Wijayatilake DS, Perlas A, et al. Practice patterns related to block selection, nerve localization and risk disclosure: a survey of the American Society of regional Anesthesia and Pain Medicine. Reg Anesth Pain Med. 2008;33:395-403.

10. Kaur A, Singh RB, Tripathi RK, Choubey S. Comparision between bupivacaine and ropivacaine in patients undergoing forearm surgeries under axillary brachial plexus block: a prospective randomized study. JCDR. 2015;9:UC01-6.

11. Maga JM, Cooper L, Gebhard RE. Outpatient regional anesthesia for upper extremity surgery update (2005 to present) distal to shoulder. Int Anesthesiol Clin. 2012;50:4755.

12. Tran DQ, Munoz L, Russo G, Finlayson R.A trick shot to the corner pocket. Reg Anesth Pain Med. 2008;33:503-4.

13. Cline E, Franz D, Polley RD, Maye J, Burkard J, Pellegrini J. Analgesia and effectiveness of levobupivacaine compared with ropivacaine in patients undergoing an axillary brachial plexus block. AANA journal. 2004;72:339-46.

14. Mankad PP, Makwana JC, Shah BJ. A comparative study of $0.5 \%$ ropivacaine and $0.5 \%$ levobupivacaine in supraclavicular brachial plexus block. Int J Med Sci Public Health. 2016;5:74-9.

15. Rathore A, Bhosale JP. A comparative double blinded study of levobupivacaine and ropivacaine in USG guided supraclavicular brachial plexus block. MedPulseInternational Medical Journal. 2017;4:320-4.

16. Garg A, Rawat HS, Sharma P, Kashalkar S, Aggarwal M. Comparison Between 0.5\% Levobupivacaine And 0.5\% Ropivacaine for Ultrasound Guided Supraclavicular Brachial Plexus Block In Patients Undergoing Upper Limb Orthopaedic Surgeries. Sch J App Med Sci. 2019;7:212-6.

17. Halaszynski TM. Ultrasound brachial plexus anesthesia and analgesia for upper extremity surgery: essentials of our current understanding, 2011. Curr Opin Anaesthesiol. 2011;24:581-591.

18. Mirza F, Brown AR. Ultrasound-guided regional anesthesia for procedures of the upper extremity. Anesthesiol Res Pract. 2011;2011:579824.

19. Mageswaran R, Choy YC. Comparison of $0.5 \%$ ropivacaine and $0.5 \%$ Levobupivacaine for infraclavicular brachial plexus block. Med J Malaysia. 2010;65:300-3. 
20. Deshpande JP, Ghodaki PS, Sardesai S. Comparative clinical study Between Racemic Bupivacaine and Levobupivacaine in Supraclavicular brachial plexus block. Indian journal of applied research. 2014;4:451-4.

21. Gamo K, Kuriyama K, Higuchi H, Uesugi A, Nakase T, Hamada M, Kawai $H$. Ultrasound-guided supraclavicular brachial plexus block in upper limb surgery: outcomes and ptient satisfaction. Bone Joint J. 2014;96-B:795-9.

22. Casati A, Borghi B, Fanelli G, Montone N, Rotini R, Fraschini G, Vinciguerra F, Torri G, Chelly J. Interscalene brachial plexus anesthesia and analgesia for open shoulder surgery: a randomized, double-blinded comparison between levobupivacaine and ropivacaine. Anesth Analg. 2003;96:253-9.

23. Liisanantti $\mathrm{O}$, Luukkonen $\mathrm{J}$, Rosenberg $\mathrm{PH}$. High-dose bupivacaine, Levobupivacaine and ropivacaine in axillary brachial plexus block. Acta Anaesthesiol Scand. 2004;48:601-6.
24. González-Suárez S, Pacheco M, Roigé J, Puig MM. Comparative study of ropivacaine $0.5 \%$ and levobupivacaine $0.33 \%$ in axillary brachial plexus block. Reg Anesth Pain Med. 2009;34:414-9.

25. Chauhan AP, Pandya J, Jain A. Comparison of block characteristics and postoperative analgesia of $0.5 \%$ Levobupivacaine with $0.5 \%$ Ropivacaine in ultrasound guided supraclavicular block for orthopedic forearm surgery a prospective, comparative, randomized, clinical study. Indian J Clin Anaesth. 2020;7:399-404.

26. Fusun E, Berit GC, Sinem SAK, Mehmet T, Tolga A, Lutfi Y. Comparative study of two agents in axillary brachial plexus block: Bupivacaine Vs Levobupivacaine. Smyrna Tip Dergisi. 2011:27-34.

27. Fournier $R$, Faust $A$, Chassot $O$, et al. Levobupivacaine $0.5 \%$ provides longer analgesia after sciatic nerve block using the Labat approach than the same dose of ropivacaine in foot and ankle surgery. Anesth Analg. 2010;110:1486-9.

Table 1: Distribution of study population according to age, sex, ASA grade in both the groups

\begin{tabular}{|l|l|l|l|}
\hline Groups & Levobupivacaine & Ropivacaine & -value \\
\hline Sex (Male/ Female) & $22 / 6$ & $19 / 9$ & $0.37^{\#}$ \\
\hline $\begin{array}{l}\text { Age (Years) } \\
\text { (Mean } \pm \text { SD) }\end{array}$ & $30.93 \pm 11.04$ & $31.14 \pm 11.76$ & $0.944^{\#}$ \\
\hline $\begin{array}{l}\text { ASA Status } \\
\text { (ASA 1/ ASA 2) }\end{array}$ & $18 / 10$ & $17 / 11$ & $0.77^{\#}$ \\
\hline${ }^{*}$ Significant \# Non Significant & & \\
\hline
\end{tabular}

Table 2: Onset of sensory Block Onset of Motor Block and Mean duration of analgesia in both groups

\begin{tabular}{|l|l|l|l|l|}
\hline Groups & Levobupivacaine & Ropivacaine & t-test value & p-value \\
\hline Onset of sensory Block (min) (Mean \pm SD) & $7.54 \pm 2.10$ & $8.55 \pm 2.08$ & -2.819 & $0.045^{*}$ \\
\hline Onset of motor Block (min) (Mean \pm SD) & $12.95 \pm 2.30$ & $14.07 \pm 2.22$ & -2.864 & $0.048^{*}$ \\
\hline Mean Duration of Analgesia (Mean \pm SD) & $9.98 \pm$ & $8.03 \pm$ & & \\
\hline${ }^{*}$ Significant \# Non Significant & & & \\
\hline
\end{tabular}

Table 3: Distribution of study population according to sensory block

\begin{tabular}{|c|c|c|c|c|c|c|c|}
\hline \multirow{2}{*}{$\begin{array}{l}\text { Sensory } \\
\text { Block }\end{array}$} & \multicolumn{3}{|c|}{ Levobupivacaine } & \multicolumn{3}{|c|}{ Ropivacaine } & \multirow[t]{2}{*}{$p$-value } \\
\hline & Zero & 1 & 2 & Zero & 1 & 2 & \\
\hline $5 \mathrm{~min}$ & 28 & 0 & 0 & 28 & 0 & 0 & 1.000 \\
\hline $10 \mathrm{~min}$ & 23 & 5 & 0 & 25 & 3 & 0 & 1.000 \\
\hline $15 \mathrm{~min}$ & 0 & 23 & 5 & 0 & 24 & 4 & 0.716 \\
\hline $20 \mathrm{~min}$ & 0 & 2 & 26 & 0 & 1 & 27 & 0.553 \\
\hline $25 \mathrm{~min}$ & 0 & 0 & 28 & 0 & 0 & 28 & 1.000 \\
\hline $30 \mathrm{~min}$ & 0 & 0 & 28 & 0 & 0 & 28 & 1.000 \\
\hline $35 \mathrm{~min}$ & 0 & 0 & 28 & 0 & 0 & 28 & 1.000 \\
\hline $40 \mathrm{~min}$ & 0 & 0 & 28 & 0 & 0 & 28 & 1.000 \\
\hline $45 \mathrm{~min}$ & 0 & 0 & 28 & 0 & 0 & 28 & 1.000 \\
\hline $50 \mathrm{~min}$ & 0 & 0 & 28 & 0 & 0 & 28 & 1.000 \\
\hline $55 \mathrm{~min}$ & 0 & 0 & 28 & 0 & 0 & 28 & 1.000 \\
\hline $60 \mathrm{~min}$ & 0 & 0 & 28 & 0 & 0 & 28 & 1.000 \\
\hline $90 \mathrm{~min}$ & 0 & 0 & 28 & 0 & 0 & 28 & 1.000 \\
\hline 2 hours & 0 & 0 & 28 & 0 & 0 & 28 & 1.000 \\
\hline 3 hours & 0 & 0 & 28 & 0 & 0 & 28 & 1.000 \\
\hline 4 hours & 0 & 3 & 25 & 0 & 6 & 22 & 1.000 \\
\hline 5 hours & 3 & 5 & 20 & 2 & 9 & 17 & 0.452 \\
\hline 6 hours & 4 & 10 & 14 & 8 & 10 & 10 & 0.368 \\
\hline 7 hours & 6 & 15 & 7 & 16 & 10 & 2 & $0.016^{*}$ \\
\hline 8 hours & 13 & 15 & 0 & 17 & 11 & 0 & 0.284 \\
\hline
\end{tabular}


Table 4: Distribution of study population according to motor block

\begin{tabular}{|c|c|c|c|c|c|c|c|c|c|}
\hline 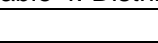 & \multicolumn{4}{|c|}{ Levobupivacaine } & \multicolumn{4}{|c|}{ Ropivacaine } & \multirow[t]{2}{*}{$p$-value } \\
\hline Motor Block & Zero & 1 & 2 & 3 & Zero & 1 & 2 & 3 & \\
\hline $5 \mathrm{~min}$ & 28 & 0 & 0 & 0 & 28 & 0 & 0 & 0 & 1.000 \\
\hline $10 \mathrm{~min}$ & 25 & 3 & 0 & 0 & 12 & 6 & 8 & 2 & $0.001^{*}$ \\
\hline $15 \mathrm{~min}$ & 5 & 18 & 5 & 0 & 2 & 10 & 6 & 10 & $0.003^{*}$ \\
\hline $20 \mathrm{~min}$ & 0 & 7 & 15 & 6 & 0 & 2 & 7 & 19 & $0.002^{*}$ \\
\hline $25 \mathrm{~min}$ & 0 & 0 & 0 & 28 & 0 & 0 & 0 & 28 & 1.000 \\
\hline $30 \mathrm{~min}$ & 0 & 0 & 0 & 28 & 0 & 0 & 0 & 28 & 1.000 \\
\hline $35 \mathrm{~min}$ & 0 & 0 & 0 & 28 & 0 & 0 & 0 & 28 & 1.000 \\
\hline 40min & 0 & 0 & 0 & 28 & 0 & 0 & 0 & 28 & 1.000 \\
\hline $45 \mathrm{~min}$ & 0 & 0 & 0 & 28 & 0 & 0 & 0 & 28 & 1.000 \\
\hline $50 \mathrm{~min}$ & 0 & 0 & 0 & 28 & 0 & 0 & 0 & 28 & 1.000 \\
\hline $55 \mathrm{~min}$ & 0 & 0 & 0 & 28 & 0 & 0 & 0 & 28 & 1.000 \\
\hline $60 \mathrm{~min}$ & 0 & 0 & 0 & 28 & 0 & 0 & 0 & 28 & 1.000 \\
\hline 90 mins & 0 & 0 & 0 & 28 & 0 & 0 & 0 & 28 & 1.000 \\
\hline 2 hours & 0 & 0 & 0 & 28 & 0 & 0 & 0 & 28 & 1.000 \\
\hline 3 hours & 0 & 0 & 0 & 28 & 0 & 1 & 3 & 24 & 1.000 \\
\hline 4 hours & 0 & 8 & 10 & 10 & 0 & 15 & 8 & 5 & 0.134 \\
\hline 5 hours & 14 & 8 & 6 & 0 & 20 & 4 & 4 & 0 & 0.248 \\
\hline 6 hours & 19 & 2 & 7 & 0 & 23 & 2 & 3 & 0 & 0.371 \\
\hline 7 hours & 25 & 3 & 0 & 0 & 28 & 0 & 0 & 0 & 0.075 \\
\hline 8 hours & 28 & 0 & 0 & 0 & 28 & 0 & 0 & 0 & 1.000 \\
\hline
\end{tabular}

Figure 1: Consort Flow Diagram

\section{Enrollment}

Allocated to intervention $(n=28)$

- Received Ropivacaine $(0.5 \%)$

- Did not receive allocated intervention $(n=0)$
Assessed for eligibility $(n=56)$

Excluded $(n=0)$

- Not meeting inclusion criteria $(n=0)$

- Declined to participate $(n=0)$

- Other reasons $(n=0)$

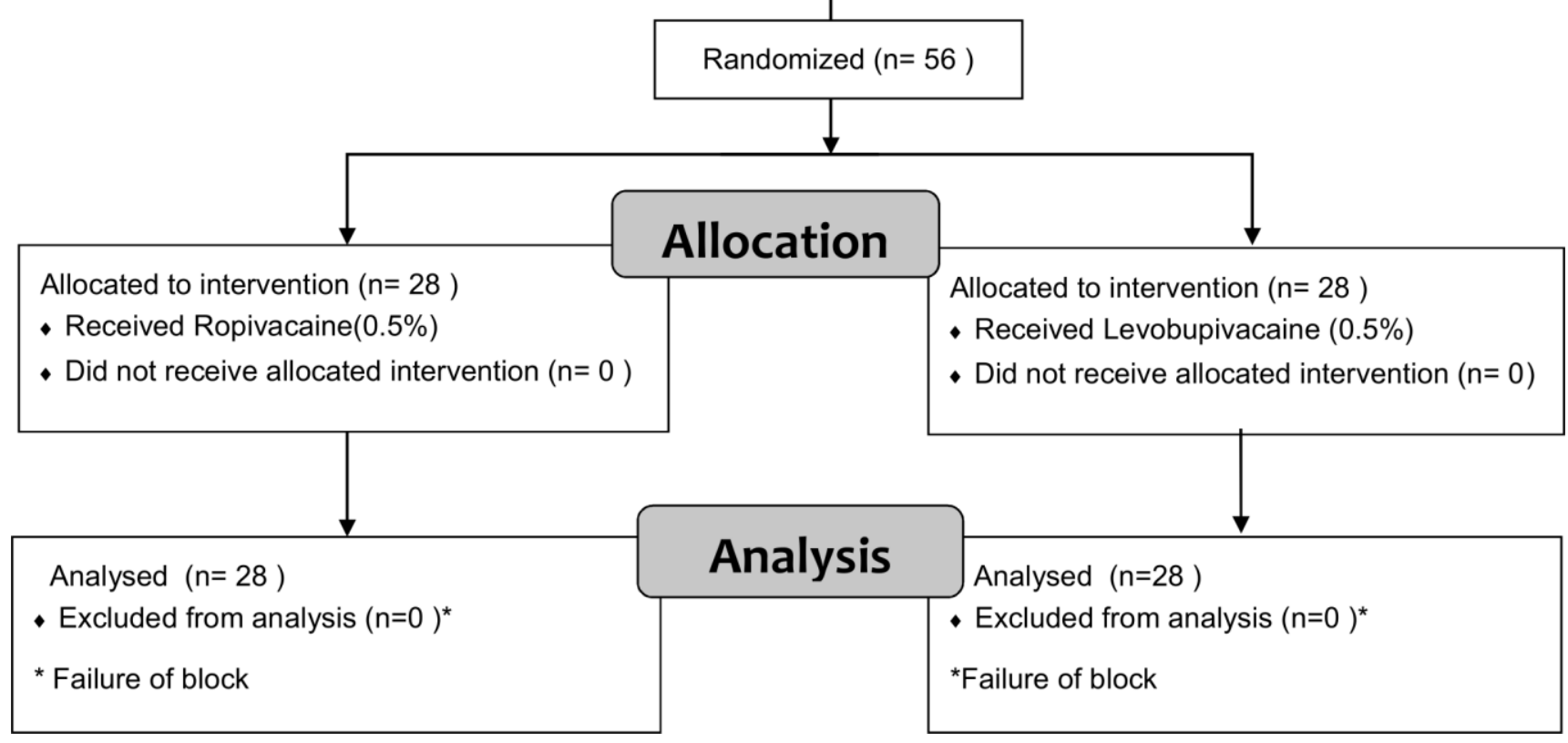


Figure - 2 Showing Hemodynamic Parameters

150

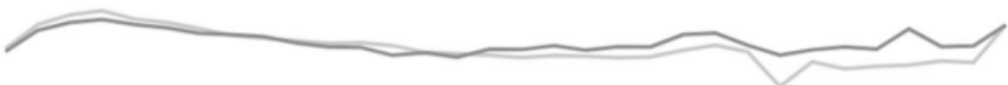

100

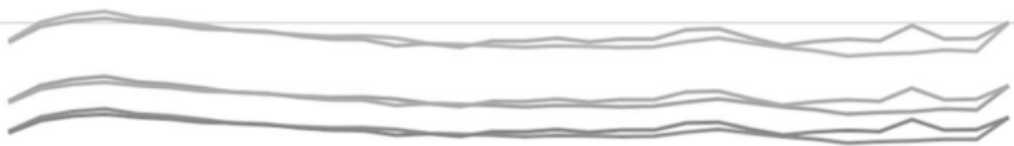

50

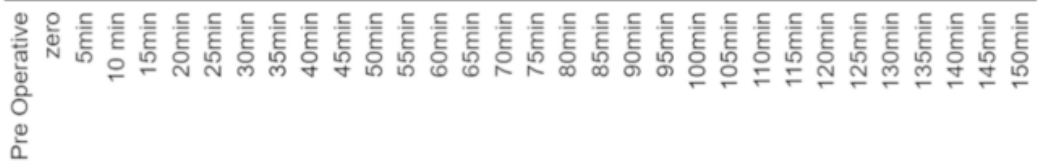

HEMODYNAMIC PARAMETERS

\section{- Heart rate Levobupivacaine \\ - Heart rate Ropivacaine \\ - SBP Levobupivacaine \\ - SBP Ropivacaine \\ - DP Levobupivacaine \\ - DP Ropivacaine \\ - MAP Levobupivacaine \\ - MAP Ropivacaine}

Figure - 3 Showing Visual Analogue Scale

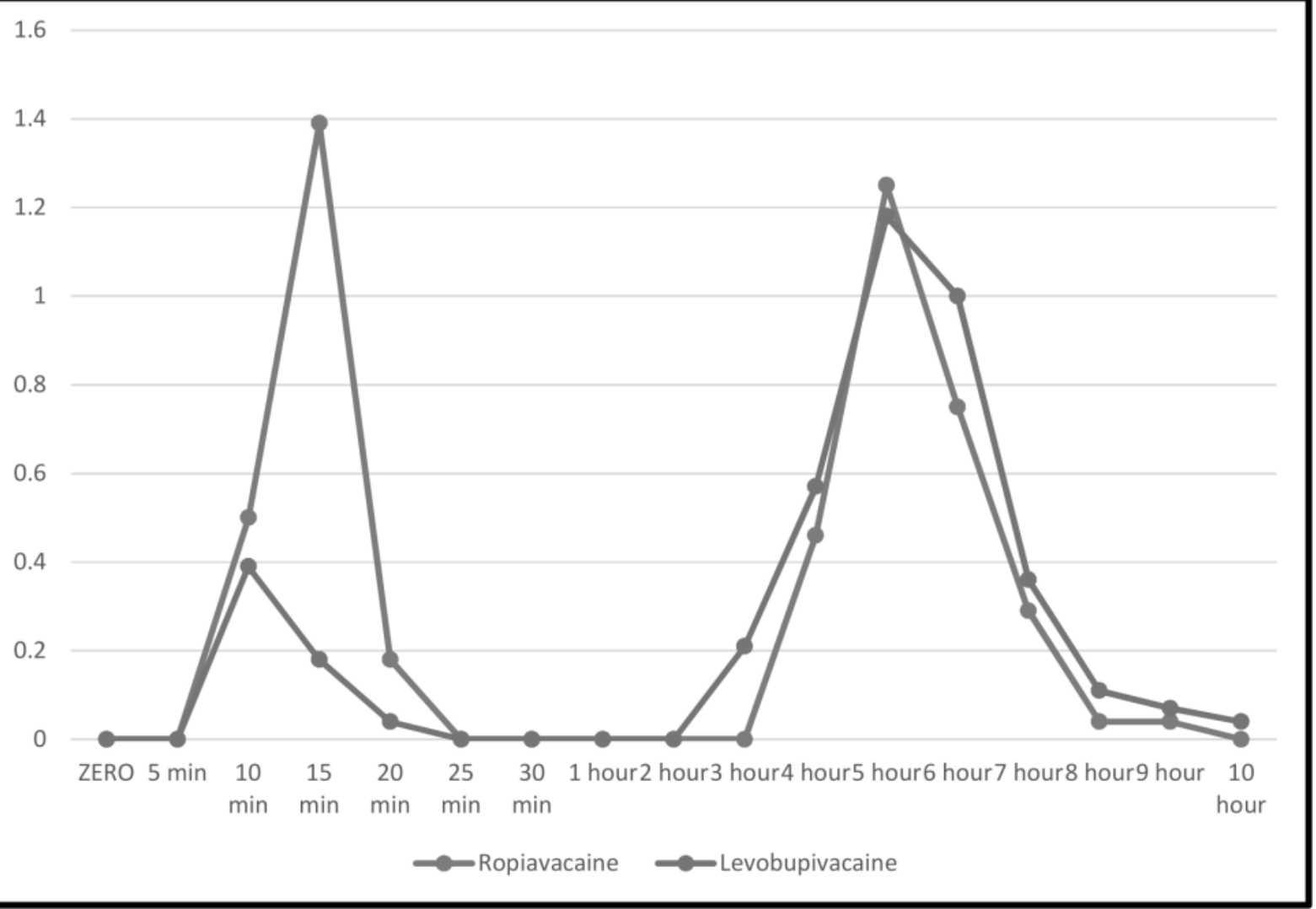

